\title{
ON BOUNDARY VALUE PROBLEMS FOR SYSTEMS OF ORDINARY, NONLINEAR, SECOND ORDER DIFFERENTIAL EQUATIONS $\left({ }^{(}\right)$
}

\author{
BY \\ PHILIP HARTMAN
}

This paper treats various problems connected with systems of differential equations of the form

$$
x^{\prime \prime}=f\left(t, x, x^{\prime}\right)
$$

for a vector $x$. The first part ( $\S 1-5)$ deals with a priori bounds for $\left|x^{\prime}\right|$ for a solution $x=x(t)$. The next part ( $\$ 8-9)$ gives existence theorems for nonsingular boundary value problems

$$
x(0)=x_{0} \text { and } x(T)=x_{T} .
$$

$\$ \$ 10-11$ give existence theorems for solutions of singular boundary value problems, that is, solutions which exist for all $t \geqq 0$ and satisfy

$$
x(0)=x_{0} \text { and }|x| \leqq R \text { for } t \geqq 0 \text { or } x \rightarrow 0 \text { as } t \rightarrow \infty .
$$

In $\S \S 12-13$, there are obtained uniqueness and continuity theorems for the solutions satisfying (2) or (3).

The results are applied in $\S \S 14-15$ to obtain existence theorems for periodic and almost periodic solutions. This application was suggested by a lecture of G. Seifert.

Finally, §§16-17 deal with existence of solutions of

$$
x^{\prime \prime}=X\left(t, x, x^{\prime}, z\right), \quad z^{\prime}=Z\left(t, x, x^{\prime}, z\right)
$$

satisfying

$$
x(0)=x_{0}, \quad x(T)=x_{T} \quad \text { and } \quad z(0)=z_{0},
$$

where $x$ and $z$ are vectors (not necessarily of the same dimension).

1. Below, if $x, f$ are vectors, $|x|$ denotes the Euclidean length of $x$ and $x \cdot f$ the scalar product of $x$ and $f$.

In $\S \S 1-5$, there will be obtained a priori bounds for the first derivatives of $n$-vector functions $x(t)$ subject to second order differential inequalities. In this direction, our results are new only for $n>1$. For reference and comparison, the following is stated for the case $n=1$.

Received by the editors December 26, 1960.

(1) This research was supported by the United States Air Force through the Air Force Office of Scientific Research of the Air Research and Development Command, under contract No. AF 18(603)-41. Reproduction in whole or in part is permitted for any purpose of the United States Government. 
Lemma 1 (Nagumo [8]). Let $\phi(s)$, where $0 \leqq s<\infty$, be a positive continuous function satisfying

$$
\int^{\infty} s d s / \phi(s)=\infty .
$$

Let $R>0$ and $T \geqq S>0$. Then there exists a constant $M$, depending only on $\phi$ and $R, S$, with the following property: If $x=x(t)$ is a real-valued function of class $C^{2}$ for $0 \leqq t \leqq T$ satisfying

$$
|x| \leqq R, \quad\left|x^{\prime \prime}\right| \leqq \phi\left(\left|x^{\prime}\right|\right),
$$

then $\left|x^{\prime}\right| \leqq M$ for $0 \leqq t \leqq T$.

For example, $M$ can be chosen to be the solution of the equation

$$
\int_{2 R / S}^{M} s d s / \phi(s)=2 R
$$

(Actually, the condition (1.1) on $\phi$ can be relaxed to the assumption that equation (1.3) have a solution $M$.)

Functions $\phi(s)$ satisfying the conditions of Lemma 1 will be called Nagumo functions. (For example, $\phi(s)=\gamma s^{2}+C>0$, where $\gamma, C$ are constants, is a Nagumo function.)

2. An example of Heinz [5] given in connection with partial differential inequalities shows that Lemma 1 is false if $x=x(t)$ is a vector-valued function. His example is the binary vector $x(t)=(\cos p t, \sin p t)$ which satisfies $|x|=1,\left|x^{\prime}\right|=|p|$ and $\left|x^{\prime \prime}\right|=\left|x^{\prime}\right|^{2}$. Thus (1.2) holds with $R=1$ and $\phi(s)$ $=s^{2}+1$, but there is no a priori bound for arbitrary $p$.

Heinz's arguments suggest the consideration of auxiliary inequalities, different in form from (1.2), say

$$
|x| \leqq R, \quad\left|x^{\prime \prime}\right| \leqq \rho^{\prime \prime},
$$

where $\rho(t)$ is a (scalar) function of class $C^{2}$ on $0 \leqq t \leqq T$.

The desired a priori bounds for $\left|x^{\prime}\right|$, in case of a vector $x$, are given by the following lemma and its consequences.

Lemma 2. Let $\phi(s), 0 \leqq s<\infty$, be a Nagumo function. Let $\rho=\rho(t)$ be a (scalar) function of class $C^{2}$ and $0 \leqq \rho(t) \leqq K_{1}$ on $0 \leqq t \leqq T$. Let $R>0, T \geqq S>0$. Then there exists a constant $M$, depending only on $\phi(s), K_{1}, R$ and $S$, with the following property: If $x=x(t)$ is a (vector-valued) function of class $C^{2}$ on $0 \leqq t \leqq T$ satisfying (1.2) and (2.1), then $\left|x^{\prime}\right| \leqq M$ for $0 \leqq t \leqq T$.

Heinz's example above shows that condition (2.1) in $x(t)$ cannot be omitted. It will be seen below that condition (1.2) cannot be omitted either. If, however, (1.2) is omitted (and (2.1) retained), one obtains a priori bounds for $\left|x^{\prime}\right|$ on all subintervals $\mu \leqq t \leqq T-\mu$ of $0 \leqq t \leqq T$, where $0<\mu<T$; cf. $\S 3$. 
REMARK. It will be shown ( $\$ 4)$ that condition (1.2) on $x(t)$ can be omitted in Lemma 2 if $\rho(t)$ satisfies

$$
\left|\rho^{\prime}\right| \leqq \theta\left|x^{\prime}\right|+C_{1}
$$

for some constants $\theta, C_{1}$ with $0<\theta<1$.

If one chooses $\phi, \rho$ to be $\gamma s^{2}+C, \alpha|x|^{2}+K$, respectively, then Lemma 2 implies the following:

Lemma 3. Let $\alpha, \gamma, R, S, T, C, K$ be non-negative constants and $T \geqq S>0$. Then there exists a constant $M=M(\alpha, \gamma, R, S, C, K)$ with the following property: If $x=x(t)$ is of class $C^{2}$ on $0 \leqq t \leqq T$ satisfying

$$
\begin{array}{ll}
|x| \leqq R, & \left|x^{\prime \prime}\right| \leqq \gamma\left|x^{\prime}\right|^{2}+C, \\
|x| \leqq R, & \left|x^{\prime \prime}\right| \leqq \alpha r^{\prime \prime}+K, \text { where } r=|x|^{2}
\end{array}
$$

then $\left|x^{\prime}\right| \leqq M$ on $0 \leqq t \leqq T$.

Heinz's example of the binary vector $x=(\cos p t, \sin p t)$ shows that condition (2.4) cannot be omitted. It is easy to give an example of a family of (scalar) functions $x(t)$ satisfying inequalities of the form (2.4) but not of the form (2.3) and for which there is no a priori bound for $\left|x^{\prime}\right|$. To this end, let $\epsilon, p>0$ and let $x(t)=x(t ; p, \epsilon)$ be the scalar function which is $1+\epsilon p^{4}(t-1 / p)^{4}$ or 1 according as $0 \leqq t \leqq 1 / p$ or $t>1 / p$. Then $x^{\prime}$ is $4 \epsilon p^{4}(t-1 / p)^{3}$ or 0 and $x^{\prime \prime}$ is $12 \epsilon p^{4}(t-1 / p)^{2}$ or 0 according as $0 \leqq t \leqq 1 / p$ or $t>1 / p$. Since $1 \leqq x \leqq 1+\epsilon$ and $x^{\prime \prime} \geqq 0$, it is clear that (2.4) holds with $R=1+\epsilon, \alpha=1 / 2$ and $K=0$. As $x^{\prime}(0)=-4 \epsilon p$, there is no a priori bound for all $p>0$ (and $\epsilon>0$ fixed).

Note that if $\gamma R<1$, then (2.3) implies (2.4) with

$$
\alpha=\gamma / 2(1-\gamma R) \text { and } K=C /(1-\gamma R) \text {. }
$$

For since

$$
r^{\prime \prime}=2\left(x \cdot x^{\prime \prime}+\left|x^{\prime}\right|^{2}\right),
$$

(2.3) shows that $r^{\prime \prime} \geqq 2(1-\gamma R)\left|x^{\prime}\right|^{2}-2 C R$ and another application of (2.3) gives $r^{\prime \prime} \geqq 2(1-\gamma R)\left(\left|x^{\prime \prime}\right|-C\right) / \gamma-2 C R$. This inequality is equivalent to (2.4)-(2.5). Conversely, if $2 R \alpha<1$, then (2.4) implies (2.3) with

$$
\gamma=2 \alpha /(1-2 R \alpha) \text { and } C=K /(1-2 R \alpha) \text {. }
$$

It can also be remarked that if (2.3) holds and, in addition,

$$
x \cdot x^{\prime \prime} \geqq 0,
$$

then (2.4) holds with $\alpha=\gamma / 2$ and $K=C$.

In view of the remark concerning (2.5), Lemma 3 has the following consequence:

COROllary 1. Let $\gamma, R, S, T, C$ be non-negative constants subject to $\gamma R<1$ 
and $T \geqq S>0$. Then the analogue of Lemma 3 holds with an $M=M(\gamma, R, S, C)$ if condition (2.4) on $x(t)$ is omitted.

In view of Heinz's example $x=(\cos p t, \sin p t), \gamma R<1$ cannot be relaxed to $\gamma R \leqq 1$ in this assertion. (Heinz's results on partial differential inequalities involve the condition $\gamma R<1 / 2$.)

The remark concerning (2.7) and Lemma 3 (or the remark concerning (2.2) and Lemma 2) imply

Corollary 2. Let $\alpha, R, S, T, K$ be non-negative constants subject to $2 R \alpha<1$ and $T \geqq S>0$. Then the analogue of Lemma 3 holds with an $M=M(\alpha, R, S, K)$ if condition (2.3) on $x(t)$ is omitted.

For the family of functions $x=x(t ; p, \epsilon)$, mentioned after Lemma 3 , $2 R \alpha=1+\epsilon$. This shows that 1 in the inequality $2 R \alpha<1$ cannot be replaced by a larger constant.

3. A priori bound on $[\mu, T-\mu]$. It will first be shown that the inequality (2.1) implies an a priori bound for $\left|x^{\prime}\right|$ on $\mu \leqq t \leqq T-\mu$, where $0<\mu<T$.

Let $0<\mu<T$ and $0 \leqq t \leqq T-\mu$. The relations

$$
x(t+\mu)-x(t)-\mu x^{\prime}(t)=\int_{t}^{t+\mu}(t+\mu-s) x^{\prime \prime}(s) d s,
$$

$t+\mu-s \geqq 0$, and (2.1) imply that

$$
\mu\left|x^{\prime}(t)\right| \leqq 2 R+\rho(t+\mu)-\rho(t)-\mu \rho^{\prime}(t) .
$$

Hence

$$
\left|x^{\prime}(t)\right| \leqq\left(2 R+K_{1}\right) / \mu-\rho^{\prime}(t) \quad \text { for } 0 \leqq t \leqq T-\mu,
$$

where $0 \leqq \rho \leqq K_{1}$ for $|x| \leqq R$.

Replacing (3.1), for $\mu \leqq t \leqq T$, by

$$
x(t)-x(t-\mu)-\mu x^{\prime}(t)=-\int_{t-\mu}^{t}(t-\mu-s) x^{\prime \prime}(s) d s
$$

leads to

$$
\left|x^{\prime}(t)\right| \leqq\left(2 R+K_{1}\right) / \mu+\rho^{\prime}(t) \quad \text { for } \mu \leqq t \leqq T .
$$

Adding (3.2) and (3.3) gives

$$
\left|x^{\prime}(t)\right| \leqq\left(2 R+K_{1}\right) / \mu \quad \text { for } \mu \leqq t \leqq T-\mu .
$$

4. On (2.2). If, in addition to (2.1), the inequality (2.2) holds, then the choice $\mu=T / 2$ in (3.2) and (3.3) shows that, for $0 \leqq t \leqq T$,

$$
\left|x^{\prime}(t)\right| \leqq\left(M_{1}+C_{1}\right) /(1-\theta) \text {, where } M_{1}=2\left(2 R+K_{1}\right) / T .
$$

Let $M$ denote the value of $\left(M_{1}+C_{1}\right) /(1-\theta)$, when $T=S$. Since $x(t)$ is given 
on an interval of length $T \geqq S$, it follows by applying the inequality $\left|x^{\prime}\right| \leqq M$ on every subinterval of length $S$, that $M$ is the desired a priori bound.

5. Proof of Lemma 2. Let $\mu=T / 2$ in (3.2) and (3.3) and let $M_{1}=M_{1}(T)$ be the constant defined in (4.1). Then (1.2) and (3.2)-(3.3) imply that

$$
\left|x^{\prime} \cdot x^{\prime \prime}\right| / \phi\left(\left|x^{\prime}\right|\right) \leqq\left|x^{\prime}\right| \leqq M_{1} \pm \rho^{\prime}
$$

where \pm is required according as $t \geqq T / 2$ or $t \leqq T / 2$.

Defined $\Phi(s)$ by

$$
\Phi(s)=\int_{0}^{s} u d u / \phi(u)
$$

Then

$$
\left|\Phi\left(\left|x^{\prime}(t)\right|\right)-\Phi\left(\left|x^{\prime}(T / 2)\right|\right)\right|=\left|\int x^{\prime} \cdot x^{\prime \prime} d t / \phi\left(\left|x^{\prime}\right|\right)\right|,
$$

where the integral is taken over the $t$-interval with endpoints $t$ and $T / 2$. It follows, therefore, from (5.1) that the expression on the left of (5.3) is majorized by $2^{-1} M_{1} T+K_{1}$. Since $\left|x^{\prime}(T / 2)\right| \leqq M_{1}$ by the case $\mu=T / 2$ of (3.4), it follows from (5.3) that

$$
\left|x^{\prime}(t)\right| \leqq M_{2} \quad \text { on } 0 \leqq t \leqq T,
$$

where $M_{2} \equiv M_{2}(T)$ is defined by

$$
M_{2}=\Phi^{-1}\left(\frac{1}{2} M_{1} T+K_{1}+\Phi\left(M_{1}\right)\right),
$$

in terms of the inverse function $\Phi^{-1}$ of the increasing function $\Phi$. Clearly $M=M_{2}(S)$ is the desired a priori bound.

6. Below there will also be needed the following:

LEMma 4 (Scorza-Dragoni [10]). Let $g\left(t, x, x^{\prime}\right)$ be a continuous and bounded (vector-valued) function for $0 \leqq t \leqq T$ and arbitrary $\left(x, x^{\prime}\right)$. Then, for artitrary $x_{0}$ and $x_{T}$, the system of differential equations

$$
x^{\prime \prime}=g\left(t, x, x^{\prime}\right)
$$

has at least one solution $x=x(t)$ satisfying

$$
x(0)=x_{0} \text { and } \quad x(T)=x_{T} .
$$

It has been pointed out by Bass [2] that this lemma is easily derived from Schauder's fixed point theorem if one considers (6.1) as an inhomogeneous form of the linear homogeneous equation $x^{\prime \prime}=0$.

7. In the remainder of the paper, the function $\rho(t)$ in Lemma 2 will be taken to be $\rho(t)=\alpha|x|^{2}+K ; \phi(s)$ will be a Nagumo function that is, a function $\phi$ satisfying the conditions of Lemma 1 . 
In order to be able to apply Lemma 4 below, it will be convenient to have the following remark: Let $f\left(t, x, x^{\prime}\right)$ be a continuous (vector-valued) function on a set

$$
D(R, T): \quad 0 \leqq t \leqq T, \quad|x| \leqq R, \quad x^{\prime} \text { arbitrary, }
$$

and let $f$ have one or more of the following properties:

$$
\begin{gathered}
x \cdot f+\left|x^{\prime}\right|^{2}>0 \text { when } x \cdot x^{\prime}=0 \text { and }|x|>0, \\
x \cdot f+\left|x^{\prime}\right|^{2}>0 \text { when } x \cdot x^{\prime}=0 \text { and }|x|=R, \\
|f| \leqq 2 \alpha\left(x \cdot f+\left|x^{\prime}\right|^{2}\right)+K, \\
|f| \leqq \phi\left(\left|x^{\prime}\right|\right) .
\end{gathered}
$$

Let $M>0$. Then there exists a continuous, bounded function $g\left(t, x, x^{\prime}\right)$ defined for $0 \leqq t \leqq T$ and arbitrary $\left(x, x^{\prime}\right)$ with the corresponding set of properties among the following:

$$
\begin{gathered}
x \cdot g+\left|x^{\prime}\right|^{2}>0 \text { when } x \cdot x^{\prime}=0 \text { and }|x|>0, \\
x \cdot g+\left|x^{\prime}\right|^{2}>0 \text { when } x \cdot x^{\prime}=0 \text { and }|x| \geqq R, \\
|g| \leqq 2 \alpha\left(x \cdot g+\left|x^{\prime}\right|^{2}\right)+K, \\
|g| \leqq \phi\left(\left|x^{\prime}\right|\right),
\end{gathered}
$$

and, at the same time, satisfying

$$
g\left(t, x, x^{\prime}\right)=f\left(t, x, x^{\prime}\right) \text { for } 0 \leqq t \leqq T,|x| \leqq R,\left|x^{\prime}\right| \leqq M .
$$

In fact, one obtains such a $g$ as follows: Let $\delta(s)$, where $0 \leqq s<\infty$, be a scalar continuous function which satisfies

$$
\delta=1,0<\delta<1, \delta=0 \text { according as } s \leqq M, M<s<2 M, s \geqq 2 M .
$$

Put

$$
\begin{aligned}
& g\left(t, x, x^{\prime}\right)=\delta\left(\left|x^{\prime}\right|\right) f\left(t, x, x^{\prime}\right) \quad \text { on } D(T, R), \\
& g\left(t, x, x^{\prime}\right)=(R /|x|) g\left(t, R x /|x|, x^{\prime}\right) \quad \text { for }|x|>R .
\end{aligned}
$$

On $D(T, R)$, the relation

$$
x \cdot g+\left|x^{\prime}\right|^{2}=\delta\left(\left|x^{\prime}\right|\right)\left(x \cdot f+\left|x^{\prime}\right|^{2}\right)+\left(1-\delta\left(\left|x^{\prime}\right|\right)\right)\left|x^{\prime}\right|^{2}
$$

makes it clear that $g$ has the desired properties on $D(T, R)$. Furthermore, the validity of the properties for $|x|=R$ implies their validity for $|x|>R$.

Note that (7.5), (7.4), respectively, imply that a solution $x=x(t)$ of $x^{\prime \prime}=f\left(t, x, x^{\prime}\right)$ satisfies (1.2), (2.1) with $\rho=\alpha|x|^{2}+K$.

8. The next desired result in the following theorem dealing with the existence of solutions of nonlinear, nonsingular, boundary value problems (under conditions more general than those in Lemma 4) for a system 


$$
x^{\prime \prime}=f\left(t, x, x^{\prime}\right) .
$$

Theorem 1. Let $f\left(t, x, x^{\prime}\right)$ be a continuous function on $D(T, R)$ in (7.1) satisfying

$$
x \cdot f+\left|x^{\prime}\right|^{2} \geqq 0 \quad \text { if } x \cdot x^{\prime}=0 \text { and }|x|=R .
$$

In addition, let $f$ satisfy (7.4) and (7.5), where $\alpha, K$ are non-negative constants and $\phi(s)$ is a Nagumo function. Let $\left|x_{0}\right|,\left|x_{T}\right| \leqq R$. Then the system (8.1) has at least one solution $x=x(t)$ satisfying $x(0)=x_{0}, x(T)=x_{T}$.

In the case $x$ is scalar, condition (7.4) can be omitted; [8].

In Theorem 1 and the assertions below, $\cdot(7.5)$ can be omitted if $2 R \alpha<1$. Also, (7.4) can be omitted, if (7.5) is replaced by

$$
|f| \leqq \gamma\left|x^{\prime}\right|^{2}+C
$$

where $\gamma, C$ are non-negative constants and $\gamma R<1$. Cf. the remarks concerning (2.5) and (2.7) above.

Proof of Theorem 1. The proof will be given first for the case that $f$ satisfies (7.3) instead of (8.2).

Let $M$ be the constant (with $T=S$ ) occurring in Lemma 2 (where $\left.\rho=\alpha|x|^{2}+K\right)$. Let $g\left(t, x, x^{\prime}\right)$ be a continuous bounded function for $0 \leqq t \leqq T$ and arbitrary $\left(x, x^{\prime}\right)$ satisfying $\left(7.3^{\prime}\right),(7.6)$ and, correspondingly, $\left(7.4^{\prime}\right)$ $\left(7.5^{\prime}\right)$. By Lemma $4,(6.1)$ has a solution $x=x(t)$ satisfying the boundary conditions (6.2).

Condition $\left(7.3^{\prime}\right)$ means that $r=|x(t)|^{2}$ satisfies $r^{\prime \prime}>0$ if $r^{\prime}=0$ and $r \geqq R^{2}$; cf. (2.6). Hence $r(t)$ cannot have a maximum value $\geqq R^{2}$ in the interval $0<t<T$. Since $r(0)=\left|x_{0}\right|^{2}, r(T)=\left|x_{T}\right|^{2}$ satisfy $r(0), r(T) \leqq R^{2}$, it follows that $r(t) \leqq R^{2}$ (that is, $|x| \leqq R$ ) on $0 \leqq t \leqq T$. In view of $\left(7.4^{\prime}\right)-\left(7.5^{\prime}\right)$, Lemma 2 is applicable to $x(t)$. Hence, $\left|x^{\prime}(t)\right| \leqq M$ for $0 \leqq t \leqq T$.

By virtue of (7.6), it follows that $x=x(t)$ is a solution of (8.1). Hence Theorem 1 is proved provided that (7.3), rather than (8.1), is assumed.

In order to remove this proviso, note that if $\epsilon>0$, the function $f\left(t, x, x^{\prime}\right)$ $+\epsilon x$ satisfies the conditions of Theorem 1 as well as condition (7.3). It is only necessary to replace $K, \phi$ in (7.4), (7.5) by $K+\epsilon R, \phi+\epsilon R$, respectively. Hence, by what has been proved,

$$
x^{\prime \prime}=f\left(t, x, x^{\prime}\right)+\epsilon x
$$

has a solution $x=x_{\epsilon}(t)$ satisfying the boundary conditions. It is clear that $\left|x_{\epsilon}(t)\right| \leqq R$ and that, for a suitable $N$ independent of $\epsilon(<1),\left|x_{\epsilon}^{\prime}(t)\right| \leqq N$ for $0 \leqq t \leqq T$. Ascoli's selection theorem shows that there exists a sequence $\epsilon_{1}, \epsilon_{2}, \cdots$ such that $0<\epsilon_{n} \rightarrow 0$ as $n \rightarrow \infty$ and that $x(t)=\lim x_{\epsilon}(t)$, as $\epsilon=\epsilon_{n} \rightarrow 0$, exists and is a solution of (8.1) and (6.2). This completes the proof of Theorem 1.

REMARK. In Theorem 1, let (8.2) be strengthened to 


$$
x \cdot f+\left|x^{\prime}\right|^{2} \geqq 0 \quad \text { if } x \cdot x^{\prime}=0,
$$

and let

$$
x_{T}=0 .
$$

Then, for the solution $x=x(t)$ just obtained, $r=|x(t)|^{2}$ satisfies

$$
r \geqq 0, \quad r^{\prime} \leqq 0 .
$$

For if (8.4) is first replaced by (7.2), it is seen that $r(t)$ has no maximum on $0<t<T$. Hence $r(t) \leqq \max (r(0), r(T))=r(0)$. Since $r(T)=0$, the same argument applies if $0<t<T$ is replaced by any subinterval $t_{0}<t<T$. This gives (8.6) if (7.2) holds. If (8.4) holds, the proof of Theorem 1 shows that $r=\left|x_{\epsilon}(t)\right|^{2}$ satisfies (8.6). But these inequalities are not lost during the limit process $\epsilon=\epsilon_{n} \rightarrow 0$.

9. In this section, there will be proved a theorem analogous to Theorem 1 , but the assumption (8.2) will be replaced by conditions on the magnitude of $\left|x_{0}\right|,\left|x_{T}\right|$ and $T$.

THEOREM 2. Let $f$ satisfy the conditions of Theorem 1 except that (8.2) need not hold. Let $x_{0}, x_{T}, R$ and $T$ be such that

$$
\beta=\max \left(\left|x_{0}\right|,\left|x_{T}\right|\right)
$$

satisfies

$$
\alpha \beta^{2}+\beta+K T^{2} / 8 \leqq R .
$$

Then (8.1) has at least one solution $x=x(t)$ satisfying $x(0)=x_{0}, x(T)=x_{T}$.

One can obtain the following assertion:

Corollary. Let $f$ be defined and continuous on $D(T, R)$ and satisfy (8.3) for some non-negative constants $\gamma, C$ such that $\gamma R<1$. Let $\beta$ in (9.1) and $T$ satisfy

$$
\gamma \beta^{2}+2(1-\gamma R) \beta+C T^{2} / 4 \leqq 2 R(1-\gamma R) .
$$

Then (8.1) has at least one solution $x=x(t)$ satisfying $x(0)=x_{0}, x(T)=x_{T}$.

Theorems 1 and 2 can be considered to be the ordinary (vector) analogue of Nagumo's results [9] for a partial (scalar) differential equation.

Proof of Theorem 2. Let $M$ be the constant supplied by Lemma 2 (with $T=S$ and $\left.\rho=\alpha|x|^{2}+K\right)$ and let $g\left(t, x, x^{\prime}\right)$ be the function supplied by $\S 7$.

By Lemma $4,(6.1)$ has a solution satisfying (6.2). Let $y=y(t)$ be the linear (vector) function satisfying

$$
y(0)=x_{0} \text { and } y(T)=x_{T}
$$

so that 


$$
x(t)=y(t)-\int_{0}^{T} G(t, s) x^{\prime \prime}(s) d s,
$$

where $T G(t, s)$ is $(T-t) s$ or $t(T-s)$ according as $0 \leqq s \leqq t \leqq T$ or $0 \leqq t \leqq s \leqq T$. By (6.1) and (7.4'), $x$ satisfies the differential inequality in (2.4). Thus $G \geqq 0$ implies

$$
|x(t)| \leqq|y(t)|+\int_{0}^{T} G(t, s)\left(\alpha r^{\prime \prime}(s)+K\right) d s .
$$

In this inequality, $r^{\prime \prime}$ can be replaced by $(r-u)^{\prime \prime}$, where $u=u(t)$ is the linear function determined by

$$
u(0)=r(0)=\left|x_{0}\right|^{2}, \quad u(T)=r(T)=\left|x_{T}\right|^{2} .
$$

Thus, by (9.5),

$$
|x(t)| \leqq|y(t)|+\alpha(u(t)-r(t))+2^{-1} K(T-t) t .
$$

Since $|y(t)| \leqq \max \left(\left|x_{0}\right|,\left|x_{T}\right|\right)=\beta, u(t) \leqq \max \left(\left|x_{0}\right|^{2},\left|x_{T}\right|^{2}\right)=\beta^{2}$ and $r \geqq 0$,

$$
|x(t)| \leqq \beta+\alpha \beta^{2}+K T^{2} / 8 \text {. }
$$

By condition (9.3), $|x(t)| \leqq R$ for $0 \leqq t \leqq T$. Also, $\left|x^{\prime}(t)\right| \leqq M$ by Lemma 2 . It follows from (7.6) that $x=x(t)$ is a solution of (8.1). This proves Theorem 2 .

10. In some of the theorems to follow, the bounded interval $0 \leqq t \leqq T$ is replaced by $0 \leqq t<\infty$.

TheOREM 3. Let $f\left(t, x, x^{\prime}\right)$ be defined and continuous on

$$
D(R): 0 \leqq t<\infty,|x| \leqq R<\infty, x^{\prime} \text { arbitrary. }
$$

For every $T>0$, let $f$ satisfy the conditions of Theorem 1 on $D(T, R)$, where the constants $\alpha, K$ and Nagumo function $\phi(s)$ which occur can depend on $T$. Then, for every $x_{0}$ in the sphere $\left|x_{0}\right| \leqq R$, there is at least one solution $x=x(t)$ of (8.1) which satisfies $x(0)=x_{0}$ and exists for $t \geqq 0$.

REMARK. If, in addition (8.4) is assumed in Theorem 3, then $r=|x(t)|^{2}$ satisfies (8.6). Also, if

$$
x \cdot f+\left|x^{\prime}\right|^{2} \geqq 0,
$$

then

$$
r \geqq 0, \quad r^{\prime} \leqq 0, \quad r^{\prime \prime} \geqq 0 .
$$

For a scalar equation in which $f$ does not depend on $x^{\prime}$, this type of theorem goes back to A. Kneser [6]; cf. [7]. For the scalar analogue of Theorem 3 in which the conditions (7.4), (7.5) on $D(T, R)$ are replaced by a Nagumo condition (7.5) alone, see [3]. For an $f$ linear in $x$ and independent of $x^{\prime}$, 
see [12]; for the general linear case, [4]. For a nonlinear system, see [2], where $f$ is subject to a majorant linear in $\left|x^{\prime}\right|$,

$$
|f| \leqq \gamma\left|x^{\prime}\right|+C
$$

on $D(T, R)$. In contrast to (10.4), Theorem 3 implies that it is sufficient to require on each $D(T, R)$ an inequality of the form (8.3) if $\gamma R<1$. There is, of course, no limitation on $R$ if $\gamma$ can be chosen arbitrarily small, that is, if

$$
f\left(t, x, x^{\prime}\right) /\left|x^{\prime}\right|^{2} \rightarrow 0 \text { as }\left|x^{\prime}\right| \rightarrow \infty
$$

uniformly for bounded $t$ and $|x| \leqq R$.

REMARK. In some of the papers just mentioned, it is assumed that

$$
x \cdot f\left(t, x, x^{\prime}\right) \geqq 0 .
$$

In this case, the conditions (7.4), (7.5) on $f$ on $D(T, R)$ are satisfied if, for example, (8.3) holds on $D(T, R)$ with arbitrary constants $\gamma=\gamma(T)>0$, $C=C(T)>0$. (A restriction of the type $\gamma R<1$ is not needed.)

Proof of Theorem 3. Let $m=1,2, \cdots$. By Theorem 1, (8.1) has a solution $x=x_{m}(t)$ on $0 \leqq t \leqq m$ satisfying $x(0)=x_{0}, x(m)=0$. Let $m \geqq T$. Then, by Lemma 2 , there is a constant $M=M_{T}$ such that $\left|x_{m}^{\prime}(t)\right| \leqq M$ for $0 \leqq t \leqq T$. Hence the sequences $\left\{x_{m}(t)\right\},\left\{x_{m}^{\prime}(t)\right\},\left\{x_{m}^{\prime \prime}(t)\right\}$, where $m \geqq T$, are uniformly bounded and equicontinuous on $0 \leqq t \leqq T$. Theorem 3 follows from Ascoli's selection theorem applied to a sequence of intervals $0 \leqq t \leqq T$, where $T \rightarrow \infty$.

The assertion concerning (8.6) follows from the Remark at the end of $\S 8$ and that concerning (10.3) follows from (2.6).

11. The next theorem gives a sufficient condition for the solutions $x=x(t)$ of (8.1) given by Theorem 3 to satisfy

$$
x(t) \rightarrow 0 \text { as } t \rightarrow \infty .
$$

THEOREM 4. Let $f\left(t, x, x^{\prime}\right)$ be defined on $D(R)$. For every number $m, 0<m<R$, let there exist a non-negative function $\sigma(t)=\sigma(t, m)$ for large $t$ satisfying

$$
\begin{gathered}
x \cdot f\left(t, x, x^{\prime}\right) \geqq \sigma(t) \geqq 0 \text { for large } t, 0<m \leqq|x| \leqq R, x^{\prime} \text { arbitrary, } \\
\qquad \int^{\infty} t \sigma(t) d t=\infty .
\end{gathered}
$$

Let $x=x(t)$ be a solution of (8.1) on $t \geqq 0$. Then (11.1) holds.

This is an analogue of (IV) in [3] dealing with scalar equations.

REMARk. Let $f\left(t, x, x^{\prime}\right)$ satisfy the conditions of Theorem 3 and, in addition, let the constants $\alpha, K$ and the function $\phi(s)$ be independent of $T$. Let $x=x(t)$ be a solution (8.1) satisfying (11.1). Then

$$
x^{\prime}(t) \rightarrow 0 \text { as } t \rightarrow \infty .
$$

For, by Lemma $2, x^{\prime}(t)$ is bounded for large $t$ and, by $(7.5), x^{\prime \prime}(t)$ is bounded 
for large $t$. The relation (11.4) then follows from the simplest Tauberian theorem (Hadamard) which states that $M_{1} \leqq \operatorname{Const}\left(M_{0} M_{2}\right)^{1 / 2}$ if $M_{0}, M_{1}, M_{2}$ are the least upper bounds for the moduli of a $C^{2}$ function and its first and second derivatives on $T \leqq t<\infty$, respectively.

Proof of Theorem 4. Let $r(t)=|x(t)|^{2}$. Since (11.2) holds for large $t, r$ satisfies $r^{\prime \prime} \geqq 0$ for large $t$. Suppose, if possible, that (11.1) fails to hold. Then there exists a constant $m$ such that $0<m \leqq r(t) \leqq R$ for large $t$. Let $\sigma(t)$ be the function belonging to the number $m$. Then

$$
q(t) \equiv 2\left(x \cdot f\left(t, x, x^{\prime}\right)+\left|x^{\prime}\right|^{2}\right) / r, \quad \text { where } \quad x=x(t) \quad \text { and } \quad r=|x(t)|^{2},
$$

satisfies

$$
q(t) \geqq 2 \sigma(t) / m \text { for large } t .
$$

Note that $r=r(t)$ satisfies the linear equation

$$
r^{\prime \prime}-q(t) r=0
$$

cf. (2.6). But the boundedness of $r(t)$ and (11.3), (11.5), (11.6) imply that

$$
r(t) \rightarrow 0 \text { as } t \rightarrow \infty
$$

(Weyl; cf., e.g., [13, pp. 601-602]). This contradicts $r \geqq m>0$ for large $t$. Hence Theorem 4 is proved.

12. This section deals with the uniqueness of solutions of (8.1) given by Theorems $1-3$. In order to obtain a uniqueness criterion, consider the linear system of differential equations

$$
y^{\prime \prime}=A(t) y+B(t) y^{\prime},
$$

where $A(t), B(t)$ are real matrices and $y$ is a vector. It is easily verified that, by virtue of (12.1),

$$
y \cdot y^{\prime \prime}+\left|y^{\prime}\right|^{2}=\left|y^{\prime}+\frac{1}{2} B^{*} y\right|^{2}+y \cdot A y-B^{*} y \cdot B^{*} y / 4,
$$

where $B^{*}$ is the transpose of $B$. Thus a sufficient condition (cf. [4]) for every solution $y=y(t)$ of (12.1) to satisfy

$$
|y(t)| \geqq 0, \quad\left(|y(t)|^{2}\right)^{\prime \prime} \geqq 0,
$$

is that

$$
4 A-B B^{*} \geqq 0,
$$

where " $Q \geqq 0$ " for a matrix $Q$ means that " $y \cdot Q y \geqq 0$ (or, equivalently, $\left.y \cdot\left(Q+Q^{*}\right) y \geqq 0\right)$ for all real vectors $y$." The fact that (12.3) implies (12.2) leads to the following uniqueness theorem:

Theorem 5. Let $f\left(t, x, x^{\prime}\right)$ be defined on $D(T, R)[$ or $D(R)]$ and possess 
continuous partial derivatives with respect to the components of $x$ and $x^{\prime}$. Let $F\left(t, x, x^{\prime}\right)$ and $G\left(t, x, x^{\prime}\right)$ denote the Jacobian matrices

$$
F\left(t, x, x^{\prime}\right)=(\partial f / \partial x), \quad G\left(t, x, x^{\prime}\right)=\left(\partial f / \partial x^{\prime}\right),
$$

and suppose that

$$
4 F-G G^{*} \geqq 0 .
$$

Then (8.1) has, at most, one solution which satisfies $x(0)=x_{0}$ and $x(T)=x_{T}$ [or which satisfies $x(0)=x_{0}$ and exists for $t \geqq 0$ ].

Of course, if an a priori bound $\left|x^{\prime}\right| \leqq M$ is known for the possible solutions involved, then (12.5) is only required for $0 \leqq t \leqq T$ [or $t \geqq 0],|x| \leqq R,\left|x^{\prime}\right| \leqq M$.

In the case that $x$ is a scalar and $f$ does not depend on $x^{\prime}$, the proof of Theorem 5 will show that the conditions on $f$ can be replaced by the assumption that $f(t, x)$ is nondecreasing in $x$ for fixed $t$.

Proof of Theorem 5. Note that if there are two such solutions $x=x_{1}(t)$ and $x=x_{2}(t)$, the difference $y=x_{2}-x_{1}$ satisfies a linear equation (12.1), where

$$
A(t)=\int_{0}^{1} F d s, \quad B(t)=\int_{0}^{1} G d s,
$$

and the argument of $F, G$ in these integrals is

$$
\left(t, s x_{2}(t)+(1-s) x_{1}(t), s x_{2}^{\prime}(t)+(1-s) x_{1}^{\prime}(t)\right) .
$$

For any (constant) vector $y$, Schwarz's inequality (applied to each component of $\left.B^{*}(t) y\right)$ gives

$$
\left|B^{*}(t) y\right|^{2} \leqq \int_{0}^{1}\left|G^{*} y\right|^{2} d s,
$$

where the argument of $G^{*}$ is (12.6). Hence

$$
y \cdot\left(4 A-B B^{*}\right) y \geqq y \cdot \int_{0}^{1}\left(4 F-G G^{*}\right) d s y ;
$$

that is, (12.3) follows from (12.5). Consequently, $y(t)=x_{2}(t)-x_{1}(t)$ satisfies (12.2).

If either $y(0)=y(T)=0$ or $y(0)=0$ and $y(t)$ exists and is bounded for $t \geqq 0$, then (12.2) implies $y(t) \equiv 0$. Hence Theorem 5 is proved.

13. This section gives a "continuity" theorem for the solutions furnished by Theorems $1-3$; namely:

Lemma 5. Let $f\left(t, x, x^{\prime}\right)$ and $f_{1}\left(t, x, x^{\prime}\right), f_{2}\left(t, x, x^{\prime}\right), \cdots$ be continuous functions defined on $D(T, R)$ [or on $D(R)]$ such that

$$
f_{n}\left(t, x, x^{\prime}\right) \rightarrow f\left(t, x, x^{\prime}\right) \text { as } n \rightarrow \infty
$$


uniformly on compact subsets of $D(T, R)$ [or of $D(R)]$. On $D(T, R)$ [or on every $D(T, R)$ ], let $f$ satisfy the conditions of Theorem 1 [with constants $\alpha, K$ and Nagumo function $\phi(s)$ depending on $T]$. Let $\left|x_{0}\right|,\left|x_{T}\right| \leqq R$. Finally, let

$$
x^{\prime \prime}=f_{n}\left(t, x, x^{\prime}\right)
$$

possess a solution $x=x_{n}(t)$ on $0 \leqq t \leqq T$ satisfying $x(0)=x_{0}, x(T)=x_{T}$ [or on $0 \leqq t<\infty$ and satisfying $\left.x(0)=x_{0}\right]$. Then there exists a sequence of positive integers $n_{1}<n_{2}<\cdots$ such that

$$
\lim _{k \rightarrow \infty} x_{n}(t)=x(t), \text { where } n=n_{k},
$$

exists uniformly on $0 \leqq t \leqq T$ [or compact subsets of $t \geqq 0$ ] and is a solution of (8.1) satisfying $x(0)=x_{0}, x(T)=x_{T}\left[\right.$ or $\left.x(0)=x_{0}\right]$.

In order to see this, consider only the case of the nonsingular boundary value problem $x(0)=x_{0}, x(T)=x_{T}$. The considerations in the singular case are similar.

Lemma 5 is an immediate consequence of Lemma 2. In fact, the inequality

$$
\left|f_{n}-f\right| \leqq 1
$$

together with the inequalities for $f$ in (7.4) and (7.5) imply that

$$
\left|f_{n}\right| \leqq 2 \alpha\left(x \cdot f_{n}+\left|x^{\prime}\right|^{2}\right)+K+1+2 R \alpha, \quad\left|f_{n}\right| \leqq \phi\left(\left|x^{\prime}\right|\right)+1 .
$$

Let $M$ denote the constant furnished by Lemma 2 , where $\rho(t), \phi(s)$ are replaced by $\alpha|x|^{2}+K+1+2 R \alpha, \phi(s)+1$, respectively.

In view of assumption (13.1), the inequality (13.4) holds for $0 \leqq t \leqq T$, $|x| \leqq R,\left|x^{\prime}\right| \leqq M$ if $n$ is sufficiently large. It follows from Lemma 2 that $\left|x^{\prime}\right| \leqq M$ for $0 \leqq t \leqq T$ and large $n$. Hence there exists a sequence of positive integers $n_{1}<n_{2}<\cdots$ such that $\lim x_{n}^{\prime}(0)$ exists as $n=n_{k} \rightarrow \infty$. Lemma 5 now follows from standard theorems.

14. Existence of periodic or almost periodic solutions is usually proved under conditions which assure that all solutions exist for $t \geqq 0$. Recently, Seifert [11] has given an existence theorem for almost periodic solutions in which this is not the case. Theorem 7 of the next section can be considered an analogue of his result for systems (even though the scalar case of Theorem 7 , without modification, does not yield Seifert's theorem which involves a differential equation of a rather special form). In this section, there will be obtained a similar theorem for the existence of periodic solutions.

Theorem 6. Let $f\left(\dot{t}, x, x^{\prime}\right)$ be defined for $-\infty<t<\infty,|x| \leqq R, x^{\prime}$ arbitrary with the properties: (i) $f$ is continuous and periodic in $t$ of period 1 for fixed $\left(x, x^{\prime}\right)$; (ii) the Jacobian matrices (12.4) exist and are continuous; (iii) $f$ satisfies (8.2); (iv) $f$ satisfies (7.4) and (7.5) with non-negative constants $\alpha, K$ and Nagumo function $\phi(s)$ independent of $t, x$; finally, (v) if $M$ is the constant sup- 
plied by Lemma 2 (with some fixed $S>0$ and $\rho(t)=\alpha|x|^{2}+K$ ), then (12.5) holds for $-\infty<t<\infty,|x| \leqq R,\left|x^{\prime}\right| \leqq M$.

Then (8.1) has at least one periodic solution of period 1. If $x=x_{1}(t)$ and $x=x_{2}(t)$ are bounded solutions for $-\infty<t<\infty$, then $\left|x_{1}(t)-x_{2}(t)\right| \equiv$ const (and const $=0$ if " $\geqq 0$ " in (12.5) is replaced by " $>0$ "). If $x=x_{1}(t)$ and $x=x_{2}(t)$ are bounded solutions for $t \geqq 0$ or $t \leqq 0$, then $\left|x_{1}(t)-x_{2}(t)\right| \leqq\left|x_{1}(0)-x_{2}(0)\right|$.

REMARK. When $x$ is a scalar, one can improve Theorem 6 slightly: condition (7.4) can be omitted in (iv); in which case, $M$ in (v) should be replaced by the $M$ supplied by Lemma 1 . If, in addition, $f\left(t, x, x^{\prime}\right)=f(t, x)$ does not depend on $x^{\prime}$, the differentiability assumptions (ii) and (v) can be replaced by the condition that $f$ is nondecreasing in $x$ for fixed $t$ (and uniqueness results if $f$ is increasing in $x$ ).

Proof of Theorem 6. By Theorems 3 and 5, the equation (8.2) has a unique solution on $t \geqq 0$ satisfying $x(0)=x_{0}$ for any $x_{0}$ in the sphere $\left|x_{0}\right| \leqq R$. Let this solution be denoted by $x=x\left(t, x_{0}\right)$. Define a map $x_{0} \rightarrow x_{1}$ of the sphere $\left|x_{0}\right| \leqq R$ into itself by putting $x_{1}=x\left(1, x_{0}\right)$. It is clear from $\left|x^{\prime}\left(t, x_{0}\right)\right| \leqq M$ and from the uniqueness of the solution $x=x\left(t, x_{0}\right)$ that the map $x_{0} \rightarrow x_{1}$ is continuous. Hence, by Brouwer's fixed point theorem, there exists a point $x_{0}=x^{*}$ such that $x\left(1, x^{*}\right)=x^{*}$.

The periodicity of $f$ implies that if $x=x(t)$ is a solution of (8.1), then $x=x(t+1)$ is also. In particular, $x\left(t+1, x_{0}\right) \equiv x\left(t, x_{1}\right)$. For the fixed point $x_{0}=x^{*}$, we have $x\left(t+1, x^{*}\right) \equiv x\left(t, x^{*}\right)$, i.e., periodicity of period 1 . This gives the existence assertion of Theorem 6 .

The "uniqueness" assertions have nothing to do with the periodicity of $f$. If $x=x_{1}(t), x_{2}(t)$ are two solutions of (8.1) for $-\infty<t<\infty$, then the proof of Theorem 5 shows that $r=\left|x_{1}-x_{2}\right|^{2}$ satisfies $r^{\prime \prime} \geqq 0$ for all $t$. But the boundedness of $r, 0 \leqq r \leqq 4 R^{2}$, implies therefore that $r(t)$ is a constant. (If " $>0$ " holds in (12.5) it is seen that $r^{\prime \prime}>0$ for some $t$ unless $r \equiv 0$.) The stability assertion concerning $\left|x_{1}-x_{2}\right|$ for $t \geqq 0$ or $t \leqq 0$ follows similarly. This completes the proof of Theorem 6 .

15. An analogous theorem for almost periodic solutions is the following:

THEOREM 7. Let $f\left(t, x, x^{\prime}\right)$ be defined for $-\infty<t<\infty,|x| \leqq R, x^{\prime}$ arbitrary with the properties (i) $f\left(t, x, x^{\prime}\right)$ is uniformly continuous for $-\infty<t<\infty$, $|x| \leqq R, x^{\prime}$ bounded and is uniformly almost periodic in $t$ for fixed $\left(x, x^{\prime}\right)$; (ii) the Jacobian matrices (12.4) exist and are uniformly bounded and uniformly continuous for $-\infty<t<\infty,|x| \leqq R, x^{\prime}$ bounded; and conditions (iii)-(v) of Theorem 6 hold.

Then (8.1) has at least one uniformly almost periodic solution.

The last parts of Theorem 6 concerning uniqueness on $-\infty<t<\infty$ and stability for $t \geqq 0$ or $t \leqq 0$ are valid here. Also, the Remark following Theorem 6 on the scalar case is applicable to Theorem 7.

Theorem 7 is a consequence of Theorem 3, the proof of Theorem 5 and 
results of Amerio [1]; cf. [1] for references to Favard. By Theorem 3, (8.1) possesses solutions $x=x(t)$ on $0 \leqq t \leqq \infty$. Also, by Lemma 2 , any solution of (8.1) satisfies $\left|x^{\prime}(t)\right| \leqq M$ if $x(t)$ exists on an interval of length $\geqq S$. In particular, $\left|x^{\prime}(t)\right| \leqq M$ on $0 \leqq t \leqq \infty$. The boundedness of $x(t)$ and $x^{\prime}(t)$ on $t \geqq 0$ for some solution implies, by [1], the existence of a solution $x=x_{1}(t)$ for $-\infty$ $<t<\infty$.

If $x=x_{1}(t), x_{2}(t)$ are two solutions of $(8.1)$ for $-\infty<t<\infty$, then, as in the proof of Theorem $6,\left|x_{1}(t)-x_{2}(t)\right| \equiv$ const.

Let $f_{1}\left(t, x, x^{\prime}\right)$ belong to the closure of the set $\left\{f\left(t+s, x, x^{\prime}\right):-\infty<s<\infty\right\}$ with respect to the sup norm for $-\infty<t<\infty,|x| \leqq R,\left|x^{\prime}\right| \leqq M$. It is clear that $f_{1}$ has properties (i)-(v) analogous to those of $f$ (if, in (i) and (ii), " $x$ ' bounded" is replaced by " $\left.\left|x^{\prime}\right| \leqq M "\right)$. Thus, if $x=x_{1}(t)$ and $x=x_{2}(t)$ are two solutions of $x^{\prime \prime}=f_{1}\left(t, x, x^{\prime}\right)$ for $-\infty<t<\infty$, then $\left|x_{1}-x_{2}\right| \equiv$ const.

It follows from [1] that if $x=x_{1}(t)$ is any solution of (8.1) on $-\infty<t<\infty$, then $x_{1}(t)$ is uniformly almost periodic. This completes the proof of Theorem 7 .

16. Let $x, z$ be vectors, not necessarily of the same dimension, and let $x^{\prime}$ be a vector of the same dimension as $x$. Let $R, Q$ be positive constants and $E$ the $\left(t, x, x^{\prime}, z\right)$-set:

$$
E: 0 \leqq t \leqq T, \quad|x| \leqq R, x^{\prime} \quad \text { arbitrary, } \quad|z| \leqq 2 Q .
$$

Let $X, Z$ be continuous vector valued functions on $E$ of the same dimension as $x, z$, respectively. The system of differential equations

$$
x^{\prime \prime}=X\left(t, x, x^{\prime}, z\right), \quad z^{\prime}=Z\left(t, x, x^{\prime}, z\right),
$$

will now be considered.

The following conditions will be imposed on $X$ :

$$
x \cdot X\left(t, x, x^{\prime}, z\right)+\left|x^{\prime}\right|^{2} \geqq 0 \text { if } x \cdot x^{\prime}=0 \text { and }|x|=R ;
$$

there exist non-negative constants $\alpha, K$ and a Nagumo function $\phi(s)$ such that

$$
|X| \leqq 2 \alpha\left(x \cdot X+\left|x^{\prime}\right|^{2}\right)+K, \quad|X| \leqq \phi\left(\left|x^{\prime}\right|\right) ;
$$

the Jacobian matrices

$$
F\left(t, x, x^{\prime}, z\right)=(\partial X / \partial x), \quad G\left(t, x, x^{\prime}, z\right)=\left(\partial X / \partial x^{\prime}\right)
$$

exist, are continuous and satisfy (12.5) on $E$.

For $Z$, it will be supposed that there exist continuous, positive functions $\sigma(t), \tau(s)$ for $0 \leqq t \leqq T, Q^{2} \leqq s \leqq(2 Q)^{2}$, respectively, satisfying

$$
\begin{gathered}
2|z \cdot Z| \leqq \\
\sigma(t) \tau\left(|z|^{2}\right) \text { for } 0 \leqq t \leqq T, Q \leqq|z| \leqq 2 Q,\left(x, x^{\prime}\right) \text { arbitrary } \\
\int_{0}^{T} \sigma(t) d t<\int_{Q^{2}}^{(2 Q)^{2}} d s / \tau(s)<\infty
\end{gathered}
$$


and that the Jacobian matrix $(\partial Z / \partial z)$ exists and is continuous on $E$.

THEOREM 8. Let $\left|x_{0}\right|,\left|x_{T}\right| \leqq R,\left|z_{0}\right| \leqq Q$. The system (16.2) has at least one solution $x=x(t), z=z(t)$ which satisfies

$$
x(0)=x_{0}, \quad x(T)=x_{T} \quad \text { and } \quad z(0)=z_{0} .
$$

It is clear that the first inequality for $X$ in (16.4) is redundant if the second is of the form $|X| \leqq \gamma\left|x^{\prime}\right|^{2}+C$ and $\gamma R<1$. It is also clear that Theorem 8 leads to an analogue of Theorem 3 .

The proof of Theorem 8 depends on Lemma 5 and on Schauder's fixed point theorem.

17. Proof of Theorem 8. Let $H$ be the Banach space of vector functions $(x(t), z(t))$ on $0 \leqq t \leqq T$ with the product topology arising from $x(t) \in C^{2}$, $z(t) \in C^{1}$. Let $M$ be the constant furnished by Lemma 2 (with $S=T$ and $\left.\rho=\alpha|x|^{2}+K\right)$ and let $N$ be a bound for $|X|,|Z|$ on the set

$$
E_{M}: 0 \leqq t \leqq T, \quad|x| \leqq R, \quad\left|x^{\prime}\right| \leqq M, \quad|z| \leqq 2 Q .
$$

Let $\omega(\epsilon)=\omega_{M}(\epsilon)$ be defined by

$$
\omega(\epsilon)=\max _{J=X, Z} \sup |\Delta J|,
$$

where $\Delta J=J\left(t, x, x^{\prime}, z\right)-J\left(t^{*}, x^{*}, x^{*}, z^{*}\right)$ and sup refers to $\left(t, x, x^{\prime}, z\right)$, $\left(t^{*}, x^{*}, x^{\prime *}, z^{*}\right)$ in $E_{M}$ and subject to $\left|t-t^{*}\right| \leqq \epsilon,\left|x-x^{*}\right| \leqq M \epsilon,\left|x^{\prime}-x^{\prime *}\right|$ $\leqq N \epsilon,\left|z-z^{*}\right| \leqq N \epsilon$.

Let $H_{0}$ be the subset of $H$ consisting of vector functions $(x(t), z(t))$ which, for $0 \leqq t \leqq T$, satisfy $|x| \leqq R,\left|x^{\prime}\right| \leqq M,\left|x^{\prime \prime}\right| \leqq N,|z| \leqq 2 Q,\left|z^{\prime}\right| \leqq N$ and

$$
\left|j(t)-j\left(t^{*}\right)\right| \leqq \omega(\epsilon) \text { if } 0 \leqq t, t^{*} \leqq T,\left|t-t^{*}\right| \leqq \epsilon \text { and } j=x^{\prime \prime}, z^{\prime},
$$

and, in addition, satisfy the boundary condition (16.8). Clearly, $H_{0}$ is a compact, convex subset of $H$.

Define a map $L: H_{0} \rightarrow H_{0}$ as follows: if $(\bar{x}(t), \bar{z}(t)) \in H_{0}$, let $L(\bar{x}(t), \bar{z}(t))$ $=(x(t), z(t))$, where $z(t)$ is defined as the unique solution of

$$
z^{\prime}=Z(t, \bar{x}(t), \bar{z}(t), z), \quad z(0)=z_{0},
$$

and $x(t)$ is the unique solution of

$$
x^{\prime \prime}=X\left(t, x, x^{\prime}, z(t)\right), \quad x(0)=x_{0} \quad \text { and } \quad x(T)=x_{T} .
$$

In order to see that $L$ is well defined, note that (17.3) defines $z(t)$ uniquely, at least for small $t \geqq 0$, since $(\partial Z / \partial z)$ exists and is continuous. Actually, $z(t)$ is defined for $0 \leqq t \leqq T$ (and satisfies $|z(t)|<2 Q$ ). For otherwise, there is a subinterval $t_{0} \leqq t \leqq t_{1}$ of $0 \leqq t \leqq T$ on which $Q \leqq|z| \leqq 2 Q$ and $\left|z\left(t_{0}\right)\right|=Q$, $\left|z\left(t_{1}\right)\right|=2 Q$. But if $s(t)=|z(t)|^{2}$, then $s^{\prime}=2 z \cdot Z(t, \bar{x}(t), \bar{z}(t), z)$ satisfies $\left|s^{\prime}\right|$ $\leqq \sigma(t) \tau(s)$. An integration of this differential inequality over the interval $t_{0} \leqq t \leqq t_{1}$ leads to 


$$
\int_{t_{0}}^{t_{1}} \sigma(t) d t \geqq \int_{Q^{2}}^{(2 Q)^{2}} d s / \tau(s),
$$

which contradicts (16.7). Also, (17.4) has a unique solution $x=x(t)$ by the existence Theorem 1 and the uniqueness Theorem 5. Finally, $(x(t), z(t))$ is in $H_{0}$.

The mapping $L: H_{0} \rightarrow H_{0}$ is continuous. In order to see this, it is sufficient to show that if $\left(\bar{x}_{n}(t), \bar{z}_{n}(t)\right), n=1,2, \cdots$, is a sequence of elements of $H_{0}$ such that $\left(\bar{x}_{n}, \bar{z}_{n}\right) \rightarrow(\bar{x}, \bar{z})$ in $H$, as $n \rightarrow \infty$, and $\left(x_{n}, z_{n}\right)=L\left(\bar{x}_{n}, \bar{z}_{n}\right)$, then $\left(x_{n}, z_{n}\right)$ $\rightarrow(x, z)=L(\bar{x}, \bar{z})$ in $H$. That $z_{n} \rightarrow z$ in $C^{1}(0, T)$ is clear from (17.3) and the uniqueness of the solution of (17.3). That $x_{n} \rightarrow x$ in $C^{2}(0, T)$ follows from (17.4) and Lemma 5.

Schauder's fixed point theorem implies that there is a point $(x(t), z(t)) \in H$ which is a fixed point of the map $L$. The point $(x(t), z(t))$ is a solution of (16.2) satisfying (16.8). This gives Theorem 8.

\section{REFERENCES}

1. L. Amerio, Soluzioni quasi-periodiche, olimitate, di sistemi differenziale non-lineari quasiperiodici, o limitati, Ann. Mat. Pura Appl. ser. 4 vol. 39 (1955) pp. 97-119.

2. R. W. Bass, On non-linear repulsive forces, Contributions to the theory of non-linear oscillations, vol. 4 (1958) pp. 201-211.

3. P. Hartman and A. Wintner, On the non-increasing solutions of $y^{\prime \prime}=f\left(x, y, y^{\prime}\right)$, Amer. J. Math. vol. 73 (1951) pp. 390-404.

4. - On disconjugate differential systems, Canad. J. Math. vol. 8 (1956) pp. 72-81.

5. E. Heinz, On certain non-linear elliptic differential equations and univalent mappings, J. Analyse Math. vol. 5 (1956/1957) pp. 197-272.

6. A. Kneser, Untersuchung und asymptotische Darstellung der Integrale gewisser Differentialgleichungen bei grossen reellen Werthen des Arguments, I, J. Reine Angew. Math. vol. 116 (1896) pp. 178-212.

7. A. Mambriani, Su un teorema relativo alle equazioni differenziali ordinarie del $2^{\circ}$ ordine, Atti della Reale Accademia Nazionale dei Lincei ser. 6 vol. 9 (1929) pp. 620-622.

8. M. Nagumo, Ueber die Differentialgleichung $y^{\prime \prime}=f\left(x, y, y^{\prime}\right)$, Proceedings of the PhysicoMathematical Society of Japan ser. 3 vol. 19 (1937) pp. 861-866.

9. - On principally linear elliptic differential equations of second order, Osaka Math. J. vol. 6 (1954) pp. 207-229.

10. G. Scorza-Dragoni, Sul problema dei valori ai limiti per $i$ systemi di equazioni differenziali del secondo ordine, Boll. Un. Mat. Ital. vol. 14 (1935) pp. 225-230.

11. G. Seifert, Almost periodic solutions for systems of differential equations near points of nonlinear first approximation, Proc. Amer. Math. Soc. vol. 11 (1960) pp. 429-435.

12. A. Wintner, On linear repulsive forces, Amer. J. Math. vol. 71 (1949) pp. 362-366.

13. - On almost free linear motions, Amer. J. Math. vol. 71 (1949) pp. 595-602.

The Johns Hopkins University, BaLtimore, Maryland 